\title{
Interference Mitigation via Joint Detection in a Fading Environment
}

\author{
Jungwon Lee \\ Mobile Solutions Lab \\ Samsung US R\&D Center \\ 4921 Directors Pl \\ San Diego, CA 92130 \\ Email: jungwon@stanfordalumni.org
}

\author{
Dimitris Toumpakaris \\ Wireless Telecommunications Laboratory \\ Department of Electrical and \\ Computer Engineering \\ University of Patras, Rio, Greece 26500 \\ Email: dtouba@upatras.gr
}

\author{
Wei Yu \\ Department of Electrical and \\ Computer Engineering \\ University of Toronto \\ Toronto, Ontario, M5S 3G4, Canada \\ Email: weiyu@comm.utoronto.ca
}

\begin{abstract}
This paper considers the detection problem for fading channels in the presence of interference when both the transmitter of interest and the interferers employ practical modulation schemes. It is shown that, for Rayleigh fading, it is possible to obtain symbol error rates that have the same dependence on the received signal-to-noise ratio as when transmission is interference-free. This is achieved using detectors that employ knowledge on the channel and on the modulation schemes of the interference. Therefore, the use of interference-aware detectors can increase the overall capacity and improve the reliability of wireless systems, justifying the use of additional knowledge on the interferers that is required for implementation as compared to detectors that do not differentiate interference from noise.
\end{abstract}

\section{INTRODUCTION}

Recently, there has been growing interest in interference mitigation in cellular communication systems. So far, the most common practice in system design has been to avoid interference using orthogonal multiplexing schemes (FDMA/TDMA/CDMA/OFDMA) and by placing cells that reuse the same frequencies far apart. Coordination among base stations allows full frequency reuse and interference mitigation using spatial equalization and, whenever possible, transmit precoding. However, in practice, it is not easy to achieve coordination among base stations in real time. In this case, if full frequency reuse is desired, transmission needs to occur in the presence of interference.

Although joint detection of the desired signal and the interference was considered in some contexts such as multiinput multi-output (MIMO) and CDMA [1-6], interference is commonly viewed as contributing to the noise floor at the receiver [7-9]. However, this is true only when the detectors do not take into account the statistics of interference. In fact, in practical systems, interference may often be less detrimental than noise of equal power, because, contrary to Gaussian noise, the signals emitted by the interfering users belong to discrete constellations. In this paper it is shown that, in such cases, the fundamental limit on the performance of the system is the power of the noise, and that discrete interference results in a loss that is bounded, irrespective of the signal-to-noise ratio.

As was shown in previous work by the authors [10], for a given channel realization, the symbol error rate (SER) curve for an interference-ignorant detector reaches an error floor as the signal-to-noise ratio (SNR) increases for a given level of signal-to-interference ratio (SIR). However, with the exception of a finite number of SIR values, no such error floor appears if a maximum-likelihood (ML) detector is employed that incorporates knowledge of the interfering channel and the statistics of the interference. Therefore, the SER has the same dependence on the SNR as when transmission is not subject to interference. In this paper it is shown that the same holds in the case where transmission is subject to Rayleigh fading: If ML detection is employed at the receiver, the SER is limited by the SNR (and not the signal-to-interference-and-noise ratio (SINR)), and its dependence on the SNR is the same as in interference-free transmission. The presence of interference does result in performance loss, but this loss is bounded by a constant factor, regardless of the SNR.

\section{SySTEM MODEL}

In this paper, a Gaussian interference channel (IC) with flat fading is considered. The signal $y[\mathrm{~m}]$ received by user 1 at time $m$ in such a channel is represented as follows:

$$
y[m]=\sum_{u=1}^{U} h_{u}[m] x_{u}[m]+z[m],
$$

where $x_{u}[m]$ is the transmit signal of user $u$ at time $m$, $h_{u}[m]$ is the channel gain from the transmitter of user $u$ to the receiver of user 1 , and $z[m]$ is the background noise. In this paper, the problem of primary interest is the symbol-bysymbol detection of $x_{1}[m]$ given $y[m]$. Thus, the time index $m$ is omitted whenever there is no potential for confusion. In vector form, (1) can be written as

$$
y=\mathbf{h}^{*} \mathbf{x}+z,
$$

where $\mathbf{h}=\left[\begin{array}{lll}h_{1}^{*} & \cdots & h_{U}^{*}\end{array}\right]^{T}$ and $\mathbf{x}=\left[\begin{array}{lll}x_{1} & \cdots & x_{U}\end{array}\right]^{T}$.

It is assumed that the transmit symbols are equiprobable and that the power of the transmit signal of user $u$ is $P_{u}=$ $\mathbb{E}\left|x_{u}\right|^{2}$. The background noise $z$ is modeled as a circularly symmetric complex Gaussian random variable with mean 0 and variance $N_{0}$, i.e., $z \sim \mathcal{C N}\left(0, N_{0}\right)$. A Rayleigh fading channel is considered. In other words, the channel gains $h_{u}$ are circularly symmetric complex Gaussian random variables with 
mean 0 and variance 1, i.e., $h_{u} \sim \mathcal{C N}(0,1)$. The variance of $h_{u}$ can be set to 1 without loss of generality by incorporating the variance of the channel gain into the transmit signal power $P_{u}$. The random variables $x_{u}$ and $h_{u}$ for $u=1, \cdots, U$ and $z$ are independent of one another. For convenience, various useful ratios can be defined in terms of $P_{u}$ and $N_{0}$ :

$$
\begin{gathered}
\mathrm{SNR} \triangleq \frac{P_{1}}{N_{0}}, \mathrm{SIR} \triangleq \frac{P_{1}}{\sum_{u=2}^{U} P_{u}}, \\
\mathrm{SINR} \triangleq \frac{P_{1}}{\sum_{u=2}^{U} P_{u}+N_{0}}, \mathrm{INR}_{u} \triangleq \frac{P_{u}}{N_{0}},
\end{gathered}
$$

For the 2-user case, INR $\mathrm{IN}_{2}$ is simply represented as INR.

This paper focuses on the fading channel scenario in which transmitters do not know the channel gains and use a fixed finite constellation (e.g. quadrature-amplitude modulation (QAM) or phase shift keying (PSK)). On the other hand, it is assumed that receiver 1 knows the channel gains as well as the modulation formats of all users.

\section{A BRief Review of Detectors}

In this section, 4 detectors, which were considered in [10], are briefly reviewed: an interference-ignorant detector, a successive interference cancellation (SIC) detector, the optimal ML detector, and the joint minimum-distance (MD) detector. The interference-ignorant detector for $x_{1}$ simply divides the received signal $y$ by the direct channel gain $h_{1}$ and then maps it to the closest point of the signal constellation of transmitter 1. On the other hand, the SIC detector performs detection for the interference first, cancels interference, and then detects the desired signal. To be more concrete, consider a two-user IC. Although the detector for receiver 1 is ultimately interested only in $x_{1}$, it can first obtain an estimate, $\hat{x}_{2}$, of $x_{2}$ by treating $x_{1}$ as noise, then detect $x_{1}$ by mapping $\frac{y-h_{2} \hat{x}_{2}}{h_{1}}$ to the closest constellation point of transmitter 1 . Note that both the interference-ignorant detector and the SIC detector assume that either the desired signal or the interference is Gaussian.

Without the Gaussian assumption on either the desired signal or the interference, the optimal ML detector for the desired signal $x_{1}$ is given as

$$
\begin{aligned}
\hat{x}_{1}(y)= & \underset{x_{1}}{\arg \max }\left[\sum_{m_{2}=0}^{M_{2}-1} \cdots \sum_{m_{U}=0}^{M_{U}-1}\right. \\
& \left.\exp \left(-\frac{\left|y-h_{1} x_{1}-\sum_{u=2}^{U} h_{u} x_{u, m_{u}}\right|^{2}}{N_{0}}\right)\right] .
\end{aligned}
$$

The optimal ML detector requires the calculation of the sum of exponential functions and the calculation of the Euclidean distance from the received signal to all combined signal constellation points. The following lower-complexity joint MD detector can be derived as an approximation of the optimal ML detector [10]:

$$
=\underset{x_{1}}{\arg \min }\left[\min _{x_{2}, \cdots, x_{U}}\left|y-h_{1} x_{1}-\sum_{u=2}^{U} h_{u} x_{u, m_{u}}\right|^{2}\right] .
$$

\section{Performance of the Detectors in a Fading INTERFERENCE CHANNEL}

In this section, the performance of the four detectors is analyzed for a fading interference channel. Here the interferenceignorant detector and the joint MD detector are of primary interest. The interference-ignorant detector has the advantage that it does not need any information on the channel gains and modulation formats of interfering users, whereas all the other detectors require such information. As is shown in [10] in the fixed channel, the joint MD detector has almost the same performance as the optimal ML detector except at very low SNR and requires much lower complexity than the optimal ML detector. Moreover, the joint MD detector significantly outperforms the SIC detector. Thus, the focus in this section is placed on the interference-ignorant detector and the joint MD detector.

In this section, it is shown that use of the joint MD detector in fading channels subject to interference guarantees that the SER can be approximated by an expression of the form $c /$ SNR, similar to channels without interference. The penalty due to the presence of interference is accounted for by the coefficient $c$, which is larger in the presence of interference, but is upper-bounded by a finite value regardless of the value of the SIR.

\section{A. SER in the Absence of Interference}

Before proceeding to the analysis in the presence of interference, the average SER is evaluated for a fading channel in the absence of interference. In this case, the received signal can be represented using the model

$$
y=h_{1} x_{1}+z,
$$

where $h_{1} \sim \mathcal{C N}(0,1), z \sim \mathcal{C N}\left(0, N_{0}\right)$, and $E\left|x_{1}\right|^{2}=P_{1}$. The average SER can be derived by considering the conditional SER given $h_{1}$ and then taking the average over the distribution of $h_{1}$ :

$$
P\left\{\hat{x}_{1} \neq x_{1}\right\}=E_{h_{1}}\left[P\left\{\hat{x}_{1} \neq x_{1} \mid h_{1}\right\}\right] .
$$

For a given $h_{1}$, the derivation of the conditional SER has been well-studied and can be found in standard digital communication books [11]. Because the exact SER expression is complicated to derive, usually, the nearest neighbor union bound (NNUB) approach is taken, where only the nearest neighbors are taken into consideration for the calculation of the SER. For the case of $M_{1}$-QAM, this NNUB approach results in the following upper bound:

$$
\begin{aligned}
& P\left\{\hat{x}_{1} \neq x_{1} \mid h_{1}\right\} \\
\leq & 4\left(1-\frac{1}{\sqrt{M_{1}}}\right) Q\left(\sqrt{\frac{3\left|h_{1}\right|^{2} \mathrm{SNR}}{M_{1}-1}}\right),
\end{aligned}
$$

where $4\left(1-1 / \sqrt{M_{1}}\right)$ represents the average number of nearest neighbors over all constellation points. It is also well known that, when $h_{1} \sim \mathcal{C N}(0,1)$ [12],

$$
E_{h_{1}}\left[Q\left(\sqrt{a\left|h_{1}\right|^{2}}\right)\right]=\frac{1}{2} q(a),
$$


where

$$
q(a)=1-\sqrt{\frac{a / 2}{1+a / 2}} .
$$

From (6), (7), and (8), the following upper bound (UB) can be obtained for the average SER

$$
p_{e, \text { no int, } \mathrm{UB}}=2\left(1-\frac{1}{\sqrt{M_{1}}}\right) q\left(\frac{3 \mathrm{SNR}}{M_{1}-1}\right) .
$$

To gain more insight on the average SER, the above upper bound is approximated to a simpler expression using the Taylor series expansion of $q(a)$ around $1 / a=0$. For large $a$,

$$
q(a) \approx 1 / a .
$$

Then the approximate upper bound (AUB) is

$$
p_{e, \text { no int,AUB }}=2\left(1-\frac{1}{\sqrt{M_{1}}}\right) \frac{M_{1}-1}{3 \text { SNR }} .
$$

Thus, the approximate upper bound decreases in proportion to the SNR as the SNR grows.

A lower bound (LB) of the average SER for $M_{1}$-QAM can be obtained by considering only half the nearest neighbors compared to the upper bound:

$$
p_{e, \text { no int }, \mathrm{LB}}=\frac{1}{2} p_{e, \text { no int,UB }} \text {. }
$$

The same approach can be applied to the approximate bound. Thus, both the approximate upper bound and the approximate lower bound have the form $c / \mathrm{SNR}$; the value of $c$ depends on whether it is used in the expression for the upper or the lower bound. Even in the presence of interference, it can be shown that the lower bound has the same form as the upper bound except that the coefficient is different. Thus, in the following, only upper bounds and approximate upper bounds are examined.

\section{B. SER for the Interference-Ignorant (II) Detector}

The interference-ignorant detector assumes no knowledge on the channel gains $h_{2}, \cdots, h_{U}$ or the modulation formats of $x_{2}, \cdots, x_{U}$. It ignores the presence of interference and makes a decision on $x_{1}$ based on the direct channel gain, $h_{1}$, and the modulation format of $x_{1}$ only. In general, the exact analysis of the interference-ignorant detector is cumbersome in the case of a fading channel. However, for the special case when all interferers employ PSK, the average SER performance can be easily analyzed. With $x_{u}$ a PSK symbol, $h_{u} x_{u}$ is a circularly symmetric complex Gaussian random variable: $h_{u} x_{u} \sim \mathcal{C N}\left(0, P_{u}\right)$. Thus the received signal can be modeled as

$$
y=h_{1} x_{1}+w,
$$

where $w \sim \mathcal{C N}\left(0, \sum_{u=2}^{U} P_{u}+N_{0}\right)$. Then the average SER, when $x_{1}$ employs $M_{1}$-QAM, is upper-bounded by

$$
p_{e, \mathrm{II}, \mathrm{UB}}=2\left(1-\frac{1}{\sqrt{M_{1}}}\right) q\left(\frac{3 \mathrm{SINR}}{M_{1}-1}\right),
$$

which, for large SINR, can be approximated by

$$
p_{e, \mathrm{II}, \mathrm{AUB}}=2\left(1-\frac{1}{\sqrt{M_{1}}}\right) \frac{M_{1}-1}{3 \mathrm{SINR}} .
$$

Therefore, the SER of the interference-ignorant detector is limited by the SINR instead of the SNR, unlike the case of no interference. In Section V, using 16-QAM as an example, it is shown by simulation that the dependence on $1 /$ SINR is maintained when QAM is employed by the interferer instead of PSK.

\section{SER for the Joint MD (JMD) Detector}

An upper bound is now derived for the SER when the joint MD detector is employed at receiver 1 . Recall that in the absence of interference, the average SER was derived by averaging the conditional SER for a given channel gain, $h_{1}$, which, in turn, is calculated using the NNUB approach. A similar approach can be employed in the presence of interference by taking the average of the conditional SER for a given $\mathbf{h}$. However, this approach is complicated because the nearest neighbors change as the channel gains vary in the presence of interference. Moreover, the combined constellation $\sum_{u=1}^{U} h_{u} x_{u}$ of the received signal is no longer square-shaped even when the constellation of each individual $x_{u}$ is squareshaped. Thus, examining the nearest neighbors does not yield a strict upper bound. Therefore, in the presence of interference, instead of considering only nearest neighbors, all the signal constellation points that can possibly result in error are considered even though the upper bound obtained in this way may not be the tightest.

With the above new approach in mind, first, the pairwise error probability (PEP) between two transmit symbol vectors is considered for a fixed $\mathbf{h}$. Then this PEP is averaged over $\mathbf{h}$, leading to a simple expression for the average PEP. Last, the upper bound of the average SER is calculated by considering all possible transmit symbol vectors and using the average PEP.

The PEP for a given $\mathbf{h}$ is derived by examining the simple detection problem where the transmit vector $\mathbf{x}$ is either $\mathbf{x}_{A}$ or $\mathbf{x}_{B}$. The maximum likelihood detector for this problem selects the transmit vector $\hat{\mathbf{x}}$ that results in $\mathbf{h}^{*} \hat{\mathbf{x}}$ closest to $y$. In this case, the PEP, i.e., the probability that $\mathrm{x}_{B}$ is erroneously detected given that $\mathbf{x}_{A}$ was transmitted is

$$
P\left\{\mathbf{x}_{A} \rightarrow \mathbf{x}_{B} \mid \mathbf{h}\right\}=Q\left(\frac{\left\|\mathbf{h}^{*}\left(\mathbf{x}_{A}-\mathbf{x}_{B}\right)\right\|}{2 \sqrt{N_{0} / 2}}\right) .
$$

The average PEP over all channel realizations is

$$
\begin{aligned}
& P\left\{\mathbf{x}_{A} \rightarrow \mathbf{x}_{B}\right\} \\
= & E_{\mathbf{h}}\left[Q\left(\sqrt{\frac{\mathbf{h}^{*}\left(\mathbf{x}_{A}-\mathbf{x}_{B}\right)\left(\mathbf{x}_{A}-\mathbf{x}_{B}\right)^{*} \mathbf{h}}{2 N_{0}}}\right)\right] .
\end{aligned}
$$

The matrix $\left(\mathbf{x}_{A}-\mathbf{x}_{B}\right)\left(\mathbf{x}_{A}-\mathbf{x}_{B}\right)^{*}$ is Hermitian. ${ }^{1}$ Since a Hermitian matrix is diagonalizable,

$$
\left(\mathbf{x}_{A}-\mathbf{x}_{B}\right)\left(\mathbf{x}_{A}-\mathbf{x}_{B}\right)^{*}=\mathbf{U} \mathbf{\Lambda} \mathbf{U}^{*}
$$

\footnotetext{
${ }^{1}$ A complex square matrix $\mathbf{A}$ is Hermitian if $\mathbf{A}^{*}=\mathbf{A}$.
} 
where $\mathbf{U}$ is a unitary matrix, i.e., $\mathbf{U}^{*} \mathbf{U}=\mathbf{U U}^{*}=\mathbf{I}$, and $\boldsymbol{\Lambda}$ is a diagonal matrix, i.e., $\boldsymbol{\Lambda}=\operatorname{diag}\left\{\lambda_{1}^{2}, \cdots, \lambda_{U}^{2}\right\}$. By defining $\tilde{\mathbf{h}}$ as $\mathbf{U}^{*} \mathbf{h}$, the average PEP can be represented as

$$
\begin{aligned}
P\left\{\mathbf{x}_{A} \rightarrow \mathbf{x}_{B}\right\} & =E_{\tilde{\mathbf{h}}}\left[Q\left(\sqrt{\frac{\tilde{\mathbf{h}}^{*} \boldsymbol{\Lambda} \tilde{\mathbf{h}}}{2 N_{0}}}\right)\right] \\
& =E_{\tilde{\mathbf{h}}}\left[Q\left(\sqrt{\frac{\sum_{u=1}^{U}\left|\tilde{h}_{i}\right|^{2} \lambda_{i}^{2}}{2 N_{0}}}\right)\right],
\end{aligned}
$$

where $\tilde{\mathbf{h}}$ has the same distribution as $\mathbf{h}$ since $h_{u}$ for $u=$ $1, \cdots, U$ are i.i.d. $\mathcal{C N}(0,1)$ and $\mathbf{U}$ is unitary. In other words, $\tilde{h}_{u}$ for $u=1, \cdots, U$ are i.i.d. $\mathcal{C N}(0,1)$.

Because $\left(\mathbf{x}_{A}-\mathbf{x}_{B}\right)\left(\mathbf{x}_{A}-\mathbf{x}_{B}\right)^{*}$ has rank 1, $\lambda_{u}$ for $u=$ $2, \cdots, U$ is 0 . It can be easily seen that $\lambda_{1}$ is the Euclidean distance $\lambda_{1}=\left\|\mathbf{x}_{A}-\mathbf{x}_{B}\right\|$ between the two transmit vectors $\mathbf{x}_{A}$ and $\mathbf{x}_{B}$. Thus, as is well known from the analysis of Rayleigh fading channels [12], the average PEP can be expressed in a closed form as

$$
P\left\{\mathbf{x}_{A} \rightarrow \mathbf{x}_{B}\right\}=\frac{1}{2} q\left(\frac{\lambda_{1}^{2}}{2 N_{0}}\right)=\frac{1}{2} q\left(\frac{\left\|\mathbf{x}_{A}-\mathbf{x}_{B}\right\|^{2}}{2 N_{0}}\right) .
$$

Using this expression for the average PEP, it is now possible to derive an upper bound for the average SER for the detection of $x_{1}$. First, the upper bound is derived for the 2-user case with both users employing QPSK, in order to simplify the description. Then, an upper bound is derived for any constellation size for 2 users. Finally, the general case of $U$ users is considered.

Because of the symmetry of QPSK, the average PEP does not depend on the transmitted symbol. Thus,

$$
P\left\{\hat{x}_{1} \neq x_{1}\right\}=P\left\{\hat{x}_{1} \neq x_{1,00} \mid \mathbf{x}=\mathbf{x}_{0000}\right\},
$$

where $\mathbf{x}_{p q r s}=\left[\begin{array}{ll}x_{1, p q} & x_{2, r s}\end{array}\right]^{T}$ and $x_{1, p q}$ and $x_{2, r s}$ are Gray-coded. In other words,

$$
x_{1, p q}=\left\{\begin{array}{cc}
\sqrt{\frac{P_{1}}{2}}(1+j), & \text { for }(p, q)=(0,0) \\
\sqrt{\frac{P_{1}}{2}}(-1+j), & \text { for }(p, q)=(0,1) \\
\sqrt{\frac{P_{1}}{2}}(-1-j), & \text { for }(p, q)=(1,1) \\
\sqrt{\frac{P_{1}}{2}}(1-j), & \text { for }(p, q)=(1,0)
\end{array} .\right.
$$

$x_{2, r s}$ can be similarly represented. The probability of error for a given $\mathbf{x}_{0000}$ is upper bounded using the average PEP:

$$
\begin{aligned}
& P\left\{\hat{x}_{1} \neq x_{1,00} \mid \mathbf{x}=\mathbf{x}_{0000}\right\} \\
\leq & \sum_{a=0}^{1} \sum_{b=0}^{1}\left[P\left\{\mathbf{x}_{0000} \rightarrow \mathbf{x}_{10 a b}\right\}+P\left\{\mathbf{x}_{0000} \rightarrow \mathbf{x}_{01 a b}\right\}\right] \\
& +P\left\{\mathbf{x}_{0000} \rightarrow \mathbf{x}_{1101}\right\}+P\left\{\mathbf{x}_{0000} \rightarrow \mathbf{x}_{1110}\right\} \\
& +P\left\{\mathbf{x}_{0000} \rightarrow \mathbf{x}_{1111}\right\}
\end{aligned}
$$

where $P\left\{\mathbf{x}_{0000} \rightarrow \mathbf{x}_{1100}\right\}$ was not included because the error event of $\mathbf{x}_{0000} \rightarrow \mathbf{x}_{1100}$ is covered by the error events of $\mathbf{x}_{0000} \rightarrow \mathbf{x}_{1000}$ and $\mathbf{x}_{0000} \rightarrow \mathbf{x}_{0100}$. Each term in the upper bound (25) can be evaluated using the average PEP (22). The squared Euclidean distance between $\mathbf{x}_{p q r s}$ and $\mathbf{x}_{0000}$ is

$$
\left\|\mathbf{x}_{\text {pqrs }}-\mathbf{x}_{0000}\right\|^{2}=d_{1, p q}^{2}+d_{2, r s}^{2},
$$

where $d_{u, 00}^{2}=0, d_{u, 01}^{2}=d_{u, 10}^{2}=2 P_{u}$, and $d_{u, 11}^{2}=4 P_{u}$ for $u=1,2$. Thus, the upper bound of the average SER is

$$
\begin{aligned}
& p_{e, 1, \mathrm{JMD}, \mathrm{UB}} \\
= & q(\mathrm{SNR})+2 q(\mathrm{SNR}+\mathrm{INR})+q(\mathrm{SNR}+2 \mathrm{INR}) \\
& +q(2 \mathrm{SNR}+\mathrm{INR})+\frac{1}{2} q(2 \mathrm{SNR}+2 \mathrm{INR}) .
\end{aligned}
$$

For sufficiently high SNR, the upper bound on the average SER of the joint MD detector can be approximated as follows:

$$
p_{e, 1, \mathrm{JMD}, \mathrm{AUB}}=f(\mathrm{SIR}) \cdot \frac{1}{\mathrm{SNR}},
$$

where

$$
f(\alpha)=1+\frac{\alpha}{2 \alpha+1}+\frac{9}{4} \cdot \frac{\alpha}{\alpha+1}+\frac{\alpha}{\alpha+2} .
$$

Hence, at sufficiently high SNR, for fixed SIR, the upper bound of the average SER is inversely proportional to SNR.

The coefficient $f(\alpha)$ in (28) is a monotonically increasing function of $\alpha$ for $\alpha \geq 0$, i.e., $f^{\prime}(\alpha)>0$ for $\alpha \geq 0$. Moreover,

$$
f(0)=1 \leq f(\alpha)<\frac{19}{4}=f(\infty)
$$

Hence, regardless of the value of SIR,

$$
P\left\{\hat{x}_{1} \neq x_{1}\right\} \lesssim \frac{19}{4 \mathrm{SNR}},
$$

This shows that the SER bound decreases in inverse proportion to the SNR. As was shown in Section IV-B, this does not hold when the interfering signal is not taken into account for detection.

As can be seen in (30), $f(\mathrm{SIR})$ in (28) is close to 1 for very strong interference and close to $19 / 4$ for almost no interference. However, the fact that $f(\mathrm{SIR})$ is an increasing function of the SIR does not necessarily imply that the actual SER increases monotonically with the SIR for a given SNR. It should be kept in mind that (28) is an upper bound for the average SER. In fact, for SIR $=\infty$, the average SER should converge to the average SER in the absence of interference:

$$
P\left\{\hat{x}_{1} \neq x_{1}\right\} \approx \frac{1}{\mathrm{SNR}} .
$$

However, the upper bound analysis in this section results in $f(\infty)=19 / 4$. This is because all the possible pairwise error events were considered for the derivation of the upper bound; these error events overlap more and more as the SIR increases. Thus, the upper bound overestimates the actual SER. Nonetheless, the upper bound analysis reveals the fact that the SER decreases in proportion to the SNR.

The above analysis sheds light on the behavior of the average SER when the user of interest employs $M_{1}$-QAM and the interferer employs any constellation of size $M_{2}$. As can be seen in (7), in the absence of interference, the average number of neighbors considered are $4\left(1-\frac{1}{\sqrt{M_{1}}}\right)$. Moreover, the neighbors considered are all $\left|h_{1}\right| d_{1, \text { min }}$ apart, where $d_{1, \text { min }}$ 
is the minimum distance of any constellation points at the transmitter. It was shown in [11] that

$$
d_{1, \min }=\sqrt{\frac{6 P_{1}}{M_{1}-1}} .
$$

On the other hand, in the presence of interference employing a constellation of size $M_{2},\left(M_{1}-1\right) M_{2}$ PEP terms are considered for the calculation of the upper bound of the average SER. ${ }^{2}$ Furthermore, in (22), the average PEP was found to be equal to

$$
P\left\{\mathbf{x}_{A} \rightarrow \mathbf{x}_{B}\right\}=\frac{1}{2} q\left(\frac{\left\|\mathbf{x}_{A}-\mathbf{x}_{B}\right\|^{2}}{2 N_{0}}\right) .
$$

The Euclidean distance between $\mathbf{x}_{A}$ and $\mathbf{x}_{B}$ for any $\mathbf{x}_{A}$ and $\mathbf{x}_{B}$ is bounded below by

$$
\left\|\mathbf{x}_{A}-\mathbf{x}_{B}\right\| \geq\left|x_{1, A}-x_{1, B}\right|,
$$

where $x_{1, A}$ and $x_{1, B}$ are the symbols of user 1 corresponding to $\mathbf{x}_{A}$ and $\mathbf{x}_{B}$, respectively. Furthermore,

$$
\left|x_{1, A}-x_{1, B}\right| \geq d_{1, \min }=\sqrt{\frac{6 P_{1}}{M_{1}-1}} .
$$

Then the average PEP bound becomes

$$
P\left\{\mathbf{x}_{A} \rightarrow \mathbf{x}_{B}\right\} \leq \frac{1}{2} q\left(\frac{3 \mathrm{SNR}}{M_{1}-1}\right),
$$

since $q(x)$ is a monotonically decreasing function of $x$. Thus,

$$
\begin{aligned}
p_{e, 1, \mathrm{JMD}, \mathrm{UB}} & =\frac{\left(M_{1}-1\right) M_{2}}{2} q\left(\frac{3 \mathrm{SNR}}{M_{1}-1}\right) \\
& \approx \frac{\left(M_{1}-1\right)^{2} M_{2}}{6 \mathrm{SNR}},
\end{aligned}
$$

for sufficiently large SNR. In the special case where $\left(M_{1}, M_{2}\right)=(4,4)$, the above bound becomes

$$
p_{e, 1, \mathrm{JMD}, \mathrm{UB}}=6 q(\mathrm{SNR}) \approx \frac{6}{\mathrm{SNR}} .
$$

This upper bound is looser than the bound given in (31) because the minimum distance was used instead of the actual distance $\left|x_{1, A}-x_{1, B}\right|$. If a tighter bound is desired, the actual distance $\left|x_{1, A}-x_{1, B}\right|$ can be calculated in a straightforward way. For example, for $M_{1}=16$, the following tighter approximate upper bound can be obtained:

$$
p_{e, 1, \mathrm{JMD}, \mathrm{UB}, 16 \mathrm{QAM}} \approx \frac{2965 M_{2}}{208 \mathrm{SNR}} \approx 14.3 \frac{M_{2}}{\mathrm{SNR}},
$$

whereas, according to (39), the bound would have been

$$
p_{e, 1, \mathrm{JMD}, \mathrm{UB}, 16 \mathrm{QAM}} \approx \frac{75 M_{2}}{2 \mathrm{SNR}}=37.5 \frac{M_{2}}{\mathrm{SNR}} .
$$

\footnotetext{
${ }^{2}$ A tighter bound can be derived by considering fewer PEP terms than $\left(M_{1}-1\right) M_{2}$. For example, in the case of $\left(M_{1}, M_{2}\right)=(4,4), 11$ terms were used in (25) instead of $\left(M_{1}-1\right) M_{2}=12$. However, the primary goal of this paper is not to derive the tightest upper bound, but to show that the SER is proportional to the SNR even in the presence of interference. As is shown in the paper, a bound using $\left(M_{1}-1\right) M_{2}$ PEP terms shows that the SER is proportional to the SNR.
}

Nonetheless, the bound (39) shows that the average SER decreases according to $c /$ SNR, where $c$ depends on the constellation sizes of the signal of interest and the interferer.

The average SER bound (39) can be compared with (10) in the absence of interference:

$$
\frac{p_{e, 1, \mathrm{JMD}, \mathrm{UB}}}{p_{e, \text { no int, UB }}}=\frac{\sqrt{M_{1}}\left(\sqrt{M_{1}}+1\right) M_{2}}{4} .
$$

In other words, the average SER in the presence of $M_{2}$-QAM interference is (approximately) at most $\frac{\sqrt{M_{1}}\left(\sqrt{M_{1}}+1\right) M_{2}}{4}$ times larger than the average SER in the absence of interference. This shows that the interference created by a signal belonging to one of $M_{2}$ constellation points increases the SER by a factor that depends on $M_{1}$ and $M_{2}$, whereas the functional dependency of SER on SNR remains the same as in the case of no interference. This is in contrast to the interference ignorant detector where the dependency of SER changes from that of SNR to that of SINR.

The SER bound for more than two users can be derived similarly. For each combined constellation point for two users, there are $\prod_{u=3}^{U} M_{u}$ possible combinations for the signals of the remaining users. Thus, the number of PEP terms that needs to be taken into account increases by $\prod_{u=3}^{U} M_{u}$ times. Thus, a simple SER bound for the $U$-user case is just the SER bound for two-user case times $\prod_{u=3}^{U} M_{u}$. Thus, the coefficient in front of $1 /$ SNR increases with the number of interferers and the constellation size. However, the coefficient would not be too large in a cellular environment because there are only one or two dominant interferers. Non-dominant interferers can be treated as part of the background noise.

Note that the average SER bound for modulation schemes other than QAM for user 1 can be calculated in a similar fashion by appropriately modifying the Euclidean distance bound (35). Then the remaining analysis can be done similarly.

\section{Simulation Results}

In this section, the performance of various detectors is evaluated by Monte Carlo simulation. The interference-ignorant detector, the SIC detector, the ordered-SIC detector, the joint MD detector, and the optimal ML detector are considered. Here the ordering for the ordered-SIC is based on the instantaneous minimum distance of user 1 and user 2's receive signal constellations. In other words, when $\left|h_{1}\right| d_{1, \min }>\left|h_{2}\right| d_{2, \min }$, then user 1 is detected first. Otherwise, user 2 is detected first.

Fig. 1 shows the performance of various detectors when 2 users employ 4-QAM and 4-QAM for SIR $=12 \mathrm{~dB}$. As can be seen in the figure, in the absence of interference, the SER curve follows the theoretical $1 /$ SNR curve quite well. The presence of interference increases the SER. However, with the use of the joint MD or optimal ML detector, the SER is approximately $f\left(10^{1.2}\right) / \mathrm{SNR} \approx 4.27 / \mathrm{SNR}$, especially for high SNR. On the other hand, the interference-ignorant detector, the SIC detector, and the ordered SIC detector fail to perform satisfactorily.

Fig. 2 shows the theoretical $f($ SIR $)$ and the actual coefficient $c$ when the SER is represented as $\mathrm{SER}=c \cdot \frac{1}{\mathrm{SNR}}$. Here, 


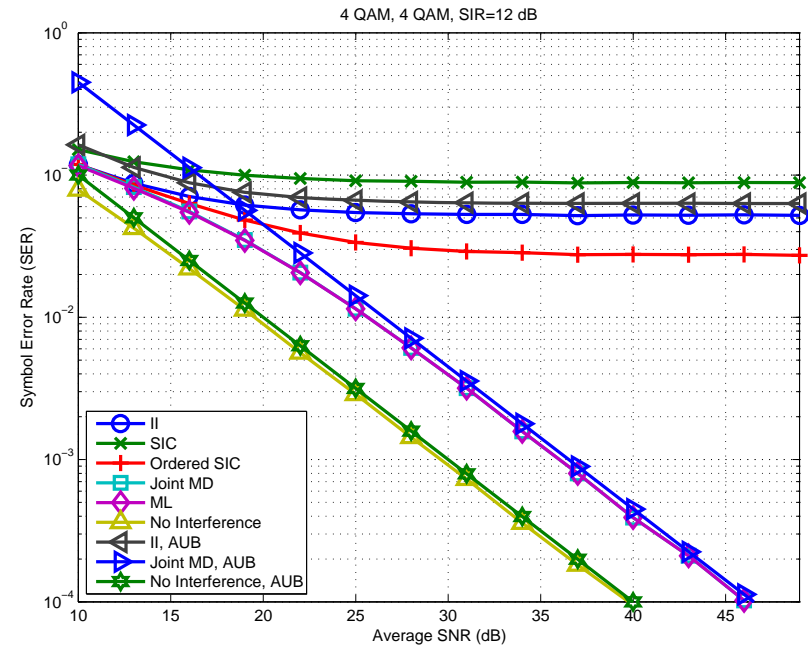

Fig. 1. Performance of various detectors with 2 users both employing 4-QAM for $\mathrm{SIR}=12 \mathrm{~dB}$

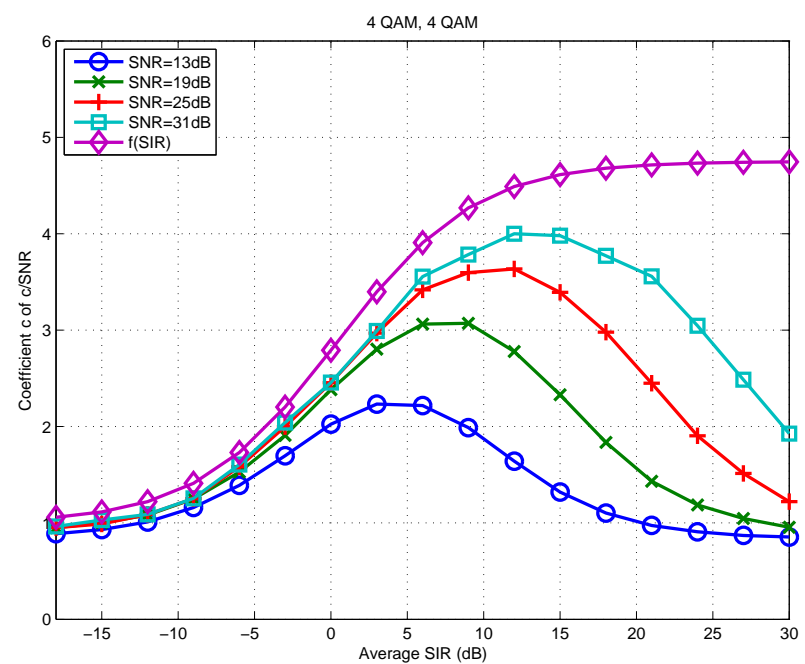

Fig. 2. SER in the presence of interference vs SER in the absence of interference when both the desired signal and the interfering signal, if present, employ 4-QAM.

the joint MD detector is used with 2 users, both employing 4QAM. The figure shows that $f(\mathrm{SIR})$ represents the SER ratio bound better for higher levels of SNR.

Figs. 3, 4, and 5 show the performance of various detectors when 2 users employ $M_{1}$-QAM and $M_{2}$-QAM with $\left(M_{1}, M_{2}\right)=(4,16),(16,4)$, and $(16,16)$. In all these figures, the simulation results match quite well with the theoretical approximate upper bounds. Here the approximate upper bound is the tighter bound that was calculated based on the actual distance of $\left|x_{1, A}-x_{1, B}\right|$ instead of the minimum distance. Comparing Fig. 1 with Fig. 3, it can be seen that the SER for the joint MD detector increases with the use of higher modulation for the interference. Similar observation can be made from the comparison of Fig. 4 and Fig. 5.

In Fig. 6, the performance of various detectors is shown when three users share the same resource and every user

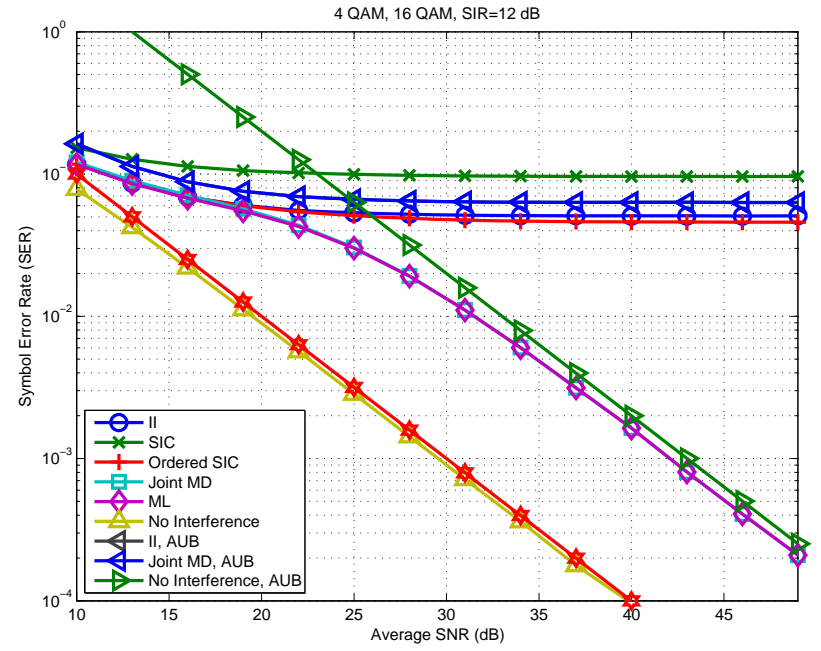

Fig. 3. Performance of various detectors with 2 users employing 4-QAM and $16 \mathrm{QAM}$ for $\mathrm{SIR}=12 \mathrm{~dB}$.

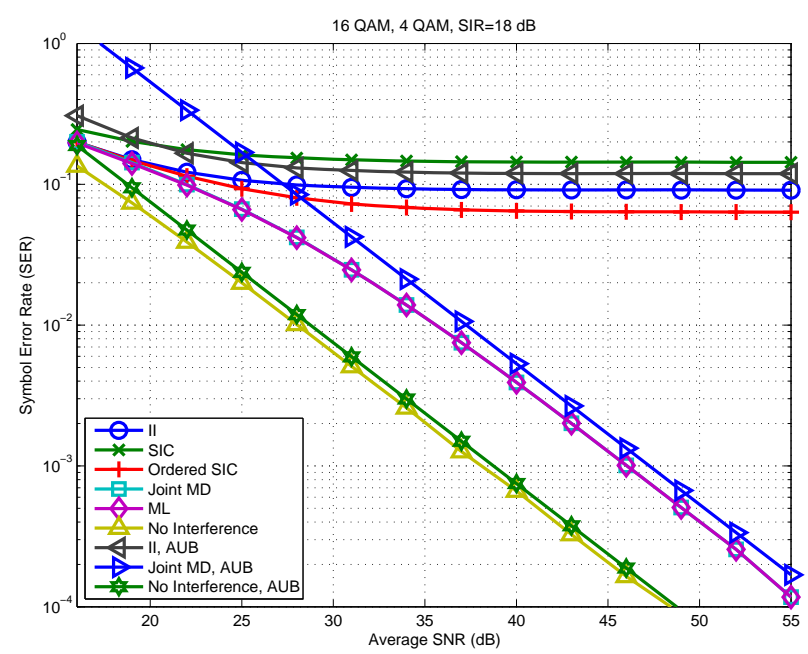

Fig. 4. Performance of various detectors with 2 users employing 16 QAM and $4-\mathrm{QAM}$ for $\mathrm{SIR}=18 \mathrm{~dB}$.

employs 4-QAM. SIR $=\frac{P_{1}}{P_{2}+P_{3}}=6 \mathrm{~dB}$. The figure shows that the actual SER follows the approximate upper bound quite well, which is given as $M_{3} f(2 \mathrm{SIR}) / \mathrm{SNR}=4 f(2 \times$ $\left.10^{0.6}\right) / \mathrm{SNR} \approx 17.07 / \mathrm{SNR}$ since $P_{1} / P_{2}=2 \mathrm{SIR}$. Moreover, it can be seen that the joint MD detector works well even in the presence of two interferers, whereas the interference-ignorant detector does not perform well.

\section{CONCLUSION}

This paper explored the detection problem for fading channels in the presence of interference. Upper bounds and approximate expressions were derived for the symbol error rate for different detection schemes. It was shown that, if no effort is made to detect the interference, the symbol error rate is limited by the SINR. On the other hand, when advanced detectors such as the joint MD detector and the optimal ML detector are employed, the symbol error rate decreases and is limited by 


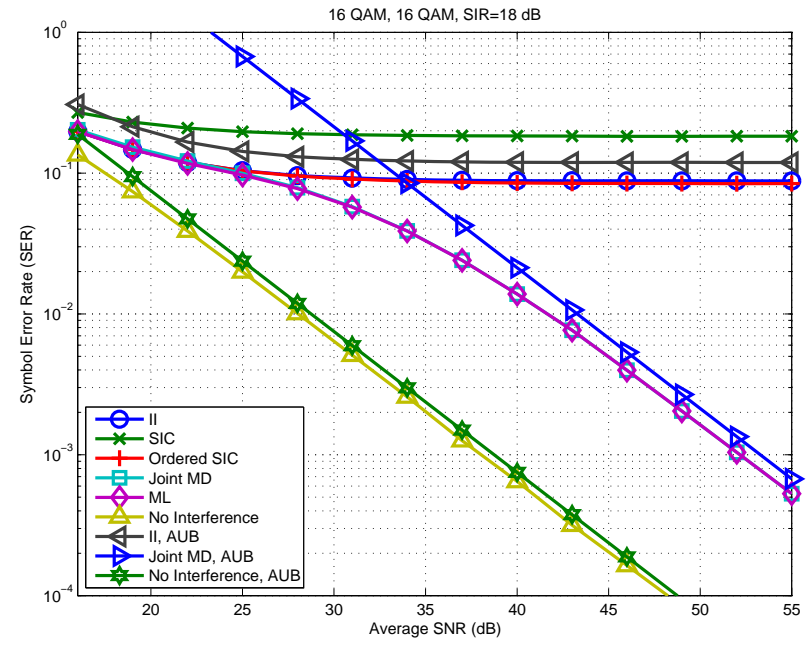

Fig. 5. Performance of various detectors with 2 users both employing 16 QAM for $\mathrm{SIR}=18 \mathrm{~dB}$.

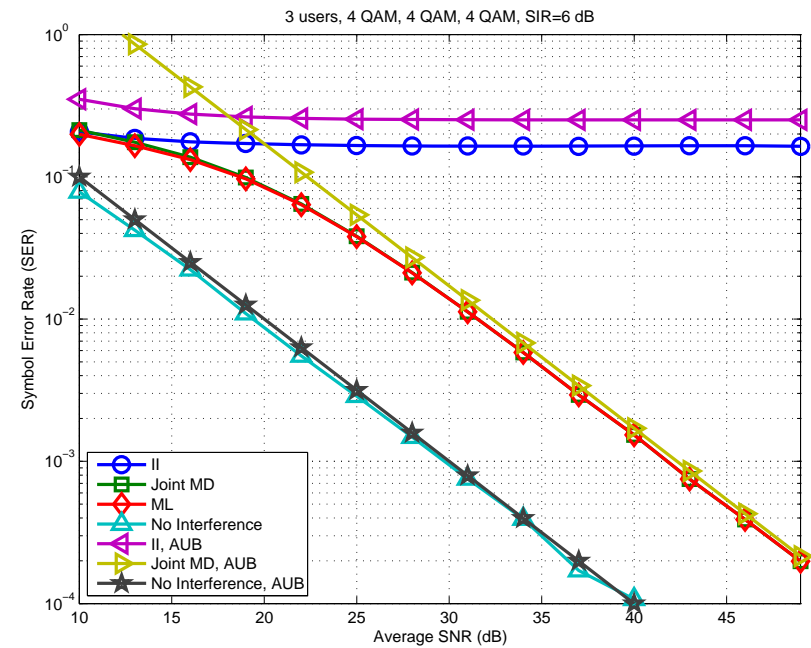

Fig. 6. Performance of various detectors with three users, all employing 4-QAM for $\mathrm{SIR}=6 \mathrm{~dB}$.

the SNR. This holds even in the presence of moderate interference, which is the most challenging scenario. Moreover, it was shown that by using advanced detectors, the difference in error rate performance of systems with interference as compared to systems shielded from interference is only a bounded constant. Therefore, although advanced detection schemes require more information about the interferers, the improved performance can make their use attractive in future communication systems.

\section{ACKNOWLEDGMENT}

Work of the second author has been performed in the framework of the FUTON-FP7-ICT-2007-215533 project, which is partially funded by the European Commission. The third author wishes to acknowledge the support of the Natural Science and Engineering Research Council (NSERC) of Canada.

\section{REFERENCES}

[1] Z. Zvonar and D. Brady, "Multiuser detection in single-path fading channels," IEEE Trans. Commun., vol. 42, no. 2/3/4, pp. 1729-1739, 1994.

[2] G. Caire, G. Taricco, J. Ventura-Traveset, and E. Biglieri, "A multiuser approach to narrowband cellular communications," IEEE Trans. Inf. Theory, vol. 43, no. 5, pp. 1503-1517, Sep. 1997.

[3] S. J. Grant and J. K. Cavers, "Further analytical results on the joint detection of cochannel signals using diversity arrays," IEEE Trans. Commun., vol. 48, no. 11, pp. 1788-1791, Nov. 2000.

[4] H. Dai and H. V. Poor, "Asymptotic spectral efficiency of multicell MIMO systems with frequency-flat fading," IEEE Trans. Signal Process., vol. 51, no. 11, pp. 2976-2988, Nov. 2003.

[5] S. J. Grant and J. K. Cavers, "System-wide capacity increase for narrowband cellular systems through multiuser detection and base station diversity arrays," IEEE Trans. Wireless Commun., vol. 3, no. 6, pp. 2072 2082, Nov. 2004.

[6] J. Kazemitabar and H. Jafarkhani, "Performance analysis of multiple antenna multi-user detection," in Information Theory and Applications Workshop, 2009.

[7] W. Yu, G. Ginis, and J. M. Cioffi, "Distributed multiuser power control for digital subscriber lines," IEEE J. Select. Areas Commun., vol. 20, pp. 1105-1115, June 2002.

[8] R. Cendrillon, W. Yu, M. Moonen, J. Verlinder, and T. Bostoen, "Optimal multi-user spectrum management for digital subscriber lines," IEEE Trans. Commun., vol. 54, pp. 922-933, May 2006.

[9] J. Lee, R. V. Sonalkar, and J. M. Cioffi, "Multi-user discrete bit-loading for DMT-based DSL systems," in IEEE Global Telecommun. Conf. (GLOBECOM), vol. 2, 2002, pp. 1259-1263.

[10] J. Lee, D. Toumpakaris, and W. Yu, "Optimal detector for discrete transmit signals in gaussian interference channels," in IEEE International Conference on Communications (ICC), 2010.

[11] J. M. Cioffi, "Course notes for digital communication: Signal processing," http://www.stanford.edu/group/cioffi/.

[12] D. Tse and P. Viswanath, Fundamentals of Wireless Communications. Cambridge Univ. Press, 2005. 